\title{
Control Strategy for Modifiable Bipedal Walking on Unknown Uneven Terrain
}

\author{
Woong-Ki Lee*, Dongkyoung Chwa* and Young-Dae Hong ${ }^{\dagger}$
}

\begin{abstract}
Previous walking pattern generation methods could generate walking patterns that allow only straight walking on flat and uneven terrain. They were unable to generate modifiable walking patterns whereby the sagittal and lateral step lengths and walking direction can be changed at every footstep. This paper proposes a novel walking pattern generation method to realize modifiable walking of humanoid robots on unknown uneven terrain. The proposed method employs a walking pattern generator based on the 3-D linear inverted pendulum model (LIPM), which enables a humanoid robot to vary its walking patterns at every footstep. A control strategy for walking on unknown uneven terrain is proposed. Virtual spring-damper (VSD) models are used to compensate for the disturbances that occur between the robot and the terrain when the robot walks on uneven terrain with unknown height. In addition, methods for generating the foot and vertical center of mass (COM) of the 3-D LIPM trajectories are developed to realize stable walking on unknown uneven terrain. The proposed method is implemented on a small-sized humanoid robot platform, DARwIn-OP and its effectiveness is demonstrated experimentally.
\end{abstract}

Keywords: Humanoid robot, Modifiable walking pattern, 3-D linear inverted pendulum model (LIPM), Uneven terrain, Virtual spring-damper (VSD) model.

\section{Introduction}

Walking pattern generation for humanoid robots is one of the most important issues in the field of humanoid research. To this end, various approaches have been proposed for generating walking patterns on flat terrain [1-5]. In addition, several methods for generating walking patterns on uneven terrain have been studied in order to realize walking of humanoid robots in real human environments; these include methods that employ stability criteria such as the zero moment point (ZMP) $[6,7]$ and contact wrench sum $[8,9]$. Moreover, attempts have been made to control the posture of a humanoid robot while it walks on uneven terrain using sensor systems [10-13]. However, the above-mentioned studies could generate walking patterns that allow only straight walking on flat and uneven terrain. They were unable to generate modifiable walking patterns whereby the sagittal and lateral step lengths and walking direction can be changed at every footstep.

In this paper, we propose a novel walking pattern generation method to realize modifiable walking of humanoid robots on unknown uneven terrain. For modifiable walking, a walking pattern generator based on the 3-D linear inverted pendulum model (LIPM) [1] is employed; it enables a humanoid robot to vary its walking period, step

$\dagger$ Corresponding Author: Dept. of Electrical and Computer Engineering, Ajou University, Korea. (ydhong@ajou.ac.kr)

* Dept. of Electrical and Computer Engineering, Ajou University, Korea. (\{yjs75311, dkchwa\}@ajou.ac.kr)

Received: March 2, 2015; Accepted: May 2, 2016 length, and walking direction independently at every footstep by adopting the ZMP variation scheme [14]. In [14], the exact height information is required to walk on uneven terrain. Therefore, a control strategy for walking on unknown uneven terrain is proposed. Virtual spring-damper (VSD) models are used to compensate for the disturbances that occur between the robot and the terrain when the robot walks on uneven terrain with unknown height. In addition, methods for generating the foot and vertical center of mass (COM) of the 3-D LIPM trajectories are developed to realize stable walking on unknown uneven terrain. The proposed method is implemented on a small-sized humanoid robot platform, DARwIn-OP, and its effectiveness is demonstrated experimentally.

The remainder of this paper is organized as follows. Section 2 describes the generation of modifiable walking patterns. In Section 3, the control strategy for walking on unknown uneven terrain is proposed. The disturbance compensation using the VSD models is explained and the methods for generating foot and vertical COM trajectories for walking on unknown uneven terrain are described. Section 4 presents and discusses the experimental results. Finally, Section 5 summarizes our findings and concludes the paper.

\section{Generation of Modifiable Walking Patterns}

To generate modifiable walking patterns, we adopt a walking pattern generation method based on the 3-D LIPM 


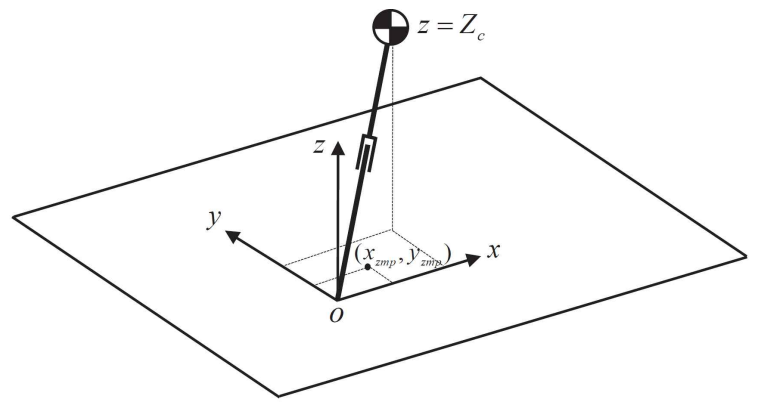

Fig. 1. 3-D LIPM

as shown in Fig. 1. From the dynamic equation of the 3-D LIPM, the sagittal and lateral COM motions of the 3-D LIPM can be obtained as follows:

with

$$
\begin{aligned}
& \mathbf{x}_{f}=\mathbf{A}(t) \mathbf{x}_{i}+\mathbf{B}_{p}(t) \\
& \mathbf{y}_{f}=\mathbf{A}(t) \mathbf{y}_{i}+\mathbf{B}_{q}(t)
\end{aligned}
$$

$$
\begin{aligned}
& \mathbf{A}(t)=\left[\begin{array}{ll}
\cosh \left(t / T_{c}\right) & \sinh \left(t / T_{c}\right) \\
\sinh \left(t / T_{c}\right) & \cosh \left(t / T_{c}\right)
\end{array}\right] \\
& \mathbf{B}_{p}(t)=-\frac{1}{T_{c}}\left[\begin{array}{l}
\sinh \left(t / T_{c}\right) * p(t) \\
\cosh \left(t / T_{c}\right) * p(t)
\end{array}\right] \\
& \mathbf{B}_{q}(t)=-\frac{1}{T_{c}}\left[\begin{array}{l}
\sinh \left(t / T_{c}\right) * q(t) \\
\cosh \left(t / T_{c}\right) * q(t)
\end{array}\right]
\end{aligned}
$$

where $T_{c}=\sqrt{Z_{c} / g}$, and $p(t)$ and $q(t)$ represent ZMP functions on the sagittal and lateral planes, respectively. * denotes the convolution operator. The sagittal and lateral COM positions and the velocities define a walking state (WS) as follows:

Definition 1: A WS is defined by

$$
\begin{aligned}
& \mathbf{x}=\left[\begin{array}{ll}
x & T_{c} v
\end{array}\right]^{T} \text { for sagittal COM motion; } \\
& \mathbf{y}=\left[\begin{array}{ll}
y & T_{c} w
\end{array}\right]^{T} \text { for lateral COM motion }
\end{aligned}
$$

where $(x, v)$ and $(y, w)$ are the COM positions and the velocities in the sagittal and lateral planes, respectively. Note that the WS is represented as a 2-D vector in each plane and that the velocity terms are multiplied by $T_{c} . \mathbf{x}_{i} / \mathbf{x}_{f}$ and $\mathbf{y}_{i} / \mathbf{y}_{f}$ in (1) are the initial/final WSs in the sagittal and lateral planes, respectively.

The first and second terms on the right-hand side of (1) are the homogeneous solution component and the particular solution component of the dynamic equation of the 3-D LIPM, respectively. In the conventional 3-D LIPM, the ZMP is fixed at the center of the foot polygon because only the homogeneous solution component is utilized. Consequently, the WS is unmodifiable throughout the single-support phase. However, the particular solution component allows the ZMP to be varied over the convex hull on the bounded foot region through the ZMP functions $p(t)$ and $q(t)$. Namely, this walking pattern generator uses real-time ZMP variation, which makes it possible to change the WS throughout the single-support phase. Consequently, it is possible to generate modifiable walking patterns without any extra footsteps to adjust for the COM motion.

For every sampling time, the footstep command, which is composed of walking period, step length, and walking direction, is entered into the walking pattern generator and then the desired WS is derived for the footstep command [14]. Next, the sagittal and lateral COM trajectories satisfying the desired WS are obtained from (1), and every leg joint trajectory is calculated by the inverse kinematics.

\section{Control Strategy for Walking on Unknown Uneven Terrain}

\subsection{Disturbance compensation}

When a humanoid robot walks on uneven terrain with unknown height, as shown in Fig. 2, a disturbance between the robot and the terrain occurs when the sole of the swing leg contacts the terrain. VSD models are used to compensate for such disturbances, as shown in Fig. 3. Two rotational VSD models are used for the $x$-axis and $y$-axis motions of the foot, and one linear VSD model is used for the $z$-axis motion of the foot. The sole of each foot is equipped with four force-sensing resistors that measure the torques, $T_{x}, T_{y}$, and the ground reaction force, $F_{z}$, on the

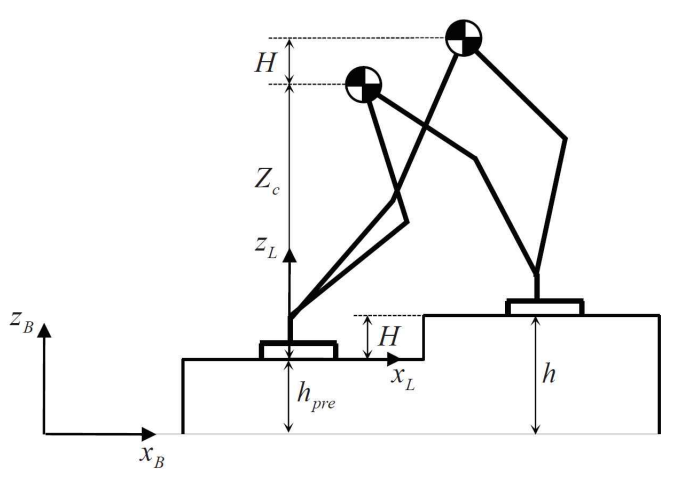

Fig. 2. Walking of humanoid robot on unknown uneven terrain

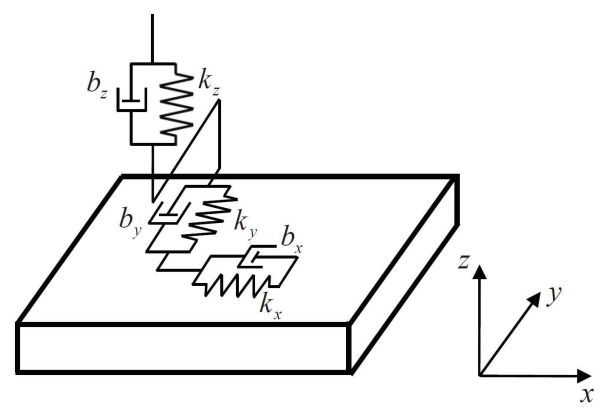

Fig. 3. VSD models for the foot 
foot. The rotational foot displacements/velocities, $\theta_{x} / \dot{\theta}_{x}$ and $\theta_{y} / \dot{\theta}_{y}$, and the linear foot displacement/velocity, $d_{z} / \dot{d}_{z}$, are calculated using the following equations:

$$
\begin{aligned}
& T_{x}=k_{x} \theta_{x}+b_{x} \dot{\theta}_{x} \\
& T_{y}=k_{y} \theta_{y}+b_{y} \dot{\theta}_{y} \\
& F_{z}=k_{z} d_{z}+b_{z} \dot{d}_{z}
\end{aligned}
$$

where $k_{x} / b_{x}, k_{y} / b_{y}$, and $k_{z} / b_{z}$ are the spring/damper coefficients.

\subsection{Generation of foot and vertical COM trajectories}

The sagittal and lateral foot trajectories of the swing leg, $x_{\text {foot }}(t)$ and $y_{\text {foot }}(t)$, are generated by cubic spline interpolation as follows:

$$
\begin{aligned}
& x_{\text {foot }}(t)=-2 \frac{S+S_{\text {pre }}}{T_{s s}{ }^{3}} t^{3}+3 \frac{S+S_{\text {pre }}}{T_{s s}{ }^{2}} t^{2}-S_{\text {pre }} \\
& y_{\text {foot }}(t)=-2 \frac{L+L_{\text {pre }}}{T_{s s}{ }^{3}} t^{3}+3 \frac{L+L_{p r e}}{T_{s s}{ }^{2}} t^{2}-L_{\text {pre }}
\end{aligned}
$$

where $S / S_{\text {pre }}$ and $L / L_{\text {pre }}$ are the sagittal and lateral step lengths from the foot of the support leg at the current/ previous footstep, respectively, and $T_{s s}$ is the single-support time. The vertical foot trajectory of the swing leg, $z_{\text {foot }}(t)$, is generated by a cycloid function. In addition, according to the commanded foot height at each footstep, the trajectory is modified by $\Delta z_{\text {foot }}(t)$, which is generated by cubic spline interpolation as follows:

$$
z_{\text {foot }}(t)=R\left\{1-\cos \left(\frac{2 \pi t}{T_{s s}}\right)\right\}+\Delta z_{\text {foot }}(t)
$$

with

$$
\Delta z_{\text {foot }}(t)=-2 \frac{H+H_{p r e}}{T_{s s}{ }^{3}} t^{3}+3 \frac{H+H_{p r e}}{T_{s s}{ }^{2}} t^{2}-H_{\text {pre }}
$$

where $H / H_{\text {pre }}$ is the foot height from the foot of the support leg at the current/previous footstep, and $R$ is the radius of the cycloid circle. Contact between the swing leg and the terrain occurs during walking on unknown uneven terrain. At this moment, the commanded foot height, $H$, at this footstep is modified for walking on uneven terrain and compensating for the disturbance between the robot and the terrain as follows:

$$
H=z_{\text {foot }}\left(t_{\text {contact }}\right)+d_{z}
$$

where $t_{\text {contact }}$ denotes the time of contact between the terrain and the sole of the swing leg. Subsequently, the foot height for the next footstep, $H_{\text {next }}$, is commanded as follows:

$$
H_{n e x t}=-h_{g}
$$

with

$$
h_{g}=h_{g}^{p r e}+H \text {. }
$$

Note that $h_{g} / h_{g}^{\text {pre }}$ is defined with respect to the base coordinate frame of the ground, as shown in Fig. 2. After the foot height of the swing leg is modified, the COM height of the 3-D LIPM should be modified to realize stable walking on uneven terrain. During the single-support phase, the COM height is maintained at a constant value, $Z_{c}$; then, it changes to $Z_{c}+H$ during the double-support phase. The vertical COM trajectory, $z(t)$, is generated by cubic spline interpolation as follows:

$$
z(t)=-2 \frac{H}{T_{d s}^{3}} t^{3}+3 \frac{H}{T_{d s}^{2}} t^{2}+Z_{c}
$$

where $T_{d s}$ is the double-support time. Note that $z(t)$ is defined with respect to the local coordinate frame of the support leg.

\subsection{Overall procedure}

The overall procedure of the proposed method is summarized in Algorithm 1. The footstep command is entered into the proposed walking pattern generator at every sample time. If the walking state is the singlesupport phase, the desired WS is derived for the footstep command, and the sagittal and lateral COM trajectories satisfying the desired WS are obtained from (1). The foot trajectories of the swing leg are generated using (3), (4). When the contact between the sole of the swing leg and the terrain occurs, the foot height is modified using (5), and the foot height for the next footstep is determined by (6). If the walking state is the double-support phase, the vertical

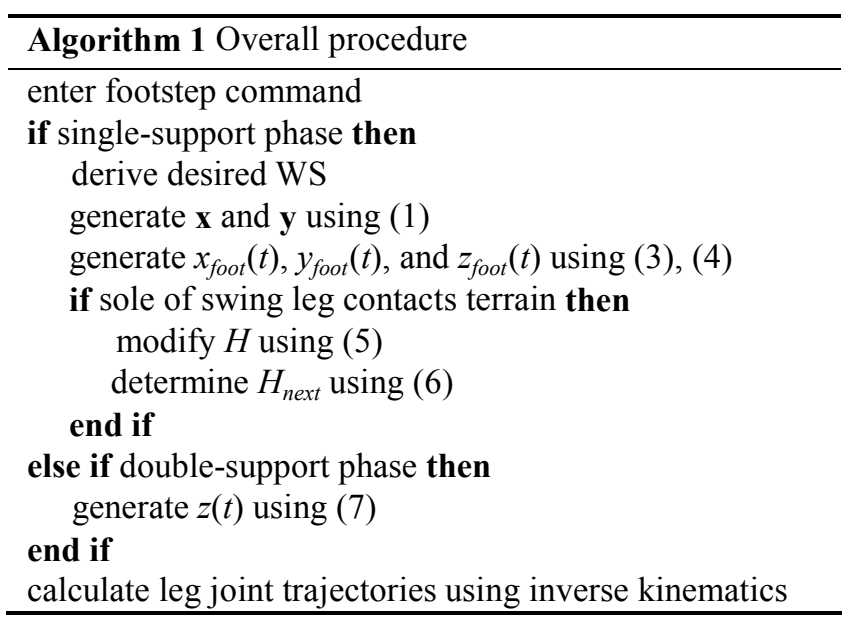


COM trajectory is generated by (7). Every leg joint trajectory is calculated by the inverse kinematics, and until the walking is terminated, these steps are repeated.

\section{Experimental Results}

The proposed method was implemented on DARwIn-OP, a small-sized humanoid robot platform (height, $45.5 \mathrm{~cm}$; weight, $2.8 \mathrm{~kg}$ ), as shown in Fig. 4 . In the experiment, the COM height, $Z_{c}$, was set as $18.35 \mathrm{~cm}$, and the radius of cycloid circle, $R$, was set as $2.0 \mathrm{~cm}$. In the experimental environment, there were five boards of different heights $(0.5,1.0$, and $1.5 \mathrm{~cm})$ and sizes, which were oriented in different directions. Table 1 lists the VSD coefficients.

Fig. 5 shows snapshots of the walking experiment on unknown uneven terrain. As shown in the figure, the humanoid robot walked stably on the unknown uneven

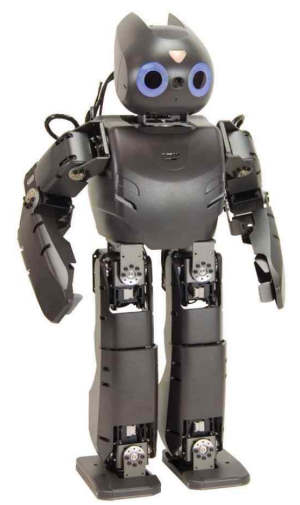

Fig. 4. Small-sized humanoid robot platform, DARwIn-OP

Table 1. VSD coefficients

\begin{tabular}{c|c|c}
\hline \multirow{2}{*}{$x$-axis VSD model } & $k_{x}$ & 100000 \\
\cline { 2 - 3 }$y$ & $b_{x}$ & 20000 \\
\hline \multirow{2}{*}{$z$-axis VSD model } & $k_{y}$ & 100000 \\
\cline { 2 - 3 } & $b_{y}$ & 20000 \\
\hline \multirow{2}{*}{$z$-axis VSD model } & $k_{z}$ & 10000 \\
\cline { 2 - 3 } & $b_{z}$ & 2000 \\
\hline
\end{tabular}

terrain by modifying the sagittal and lateral step lengths, $S$ and $L$, and the walking direction $\theta$, at every footstep. Table 2 presents the commanded modifiable walking patterns, which are predetermined or afforded by the footstep planner $[15,16]$ for walking on uneven terrain. Using the proposed method, the heights of the boards were estimated, and then, the commanded foot height, $H$, was modified at each footstep for walking on the unknown uneven terrain. As shown in Fig. 6, the sagittal and lateral COM trajectories were generated to achieve the commanded modifiable walking patterns. Moreover, the COM height was maintained at a constant value during the singlesupport phase, and during the double-support phase, it was varied according to the estimated heights of the boards to enable the robot to step up or down.

Fig. 7 shows the measured ZMP trajectories in the walking experiment. It can be seen that the ZMP trajectories followed the foot trajectories with a slight variation that was mainly due to the disturbance caused by the contact between the foot of the robot and the terrain as well as the dynamic differences between the humanoid robot and the 3-D LIPM. However, these were compensated for by the proposed method; therefore, the ZMP trajectories were within the boundaries of the foot

Table 2. Commanded modifiable walking patterns (time, length, and angle are expressed in units of seconds, centimeters, and degrees, respectively)

\begin{tabular}{c|c|c|c|c|c|c}
\hline Steps (swing leg) & $T_{s s}$ & $T_{d s}$ & $S$ & $L$ & $\theta$ & $H$ \\
\hline $1^{\text {st }}$ (right leg) & 0.80 & 0.60 & 5.00 & -7.40 & 0.00 & 0.00 \\
\hline $2^{\text {nd }}$ (left leg) & 0.80 & 0.60 & 6.00 & 7.40 & 0.00 & 0.92 \\
\hline $3^{\text {rd }}$ (right leg) & 0.80 & 0.60 & 5.00 & -7.40 & 0.00 & -0.93 \\
\hline $4^{\text {th }}$ (left leg) & 0.80 & 0.60 & 2.00 & 7.40 & 0.00 & 0.95 \\
\hline $5^{\text {th }}$ (right leg) & 0.80 & 0.60 & 5.00 & -10.40 & 0.00 & -0.93 \\
\hline $6^{\text {th }}$ (left leg) & 0.80 & 0.60 & 7.00 & 7.40 & 0.00 & 0.97 \\
\hline $7^{\text {th }}$ (right leg) & 0.80 & 0.60 & 4.00 & -7.40 & 0.00 & -0.46 \\
\hline $8^{\text {th }}$ (left leg) & 0.80 & 0.60 & 7.00 & 7.40 & 0.00 & 0.94 \\
\hline $9^{\text {th }}$ (right leg) & 0.80 & 0.60 & 5.00 & -11.40 & -20.00 & -0.47 \\
\hline $10^{\text {th }}$ (left leg) & 0.80 & 0.60 & 7.00 & 7.40 & 0.00 & -0.98 \\
\hline $11^{\text {th }}$ (right leg) & 0.80 & 0.60 & 5.00 & -7.40 & 0.00 & 0.00 \\
\hline $12^{\text {th }}$ (left leg) & 0.80 & 0.60 & 0.00 & 7.40 & 0.00 & 0.00 \\
\hline
\end{tabular}
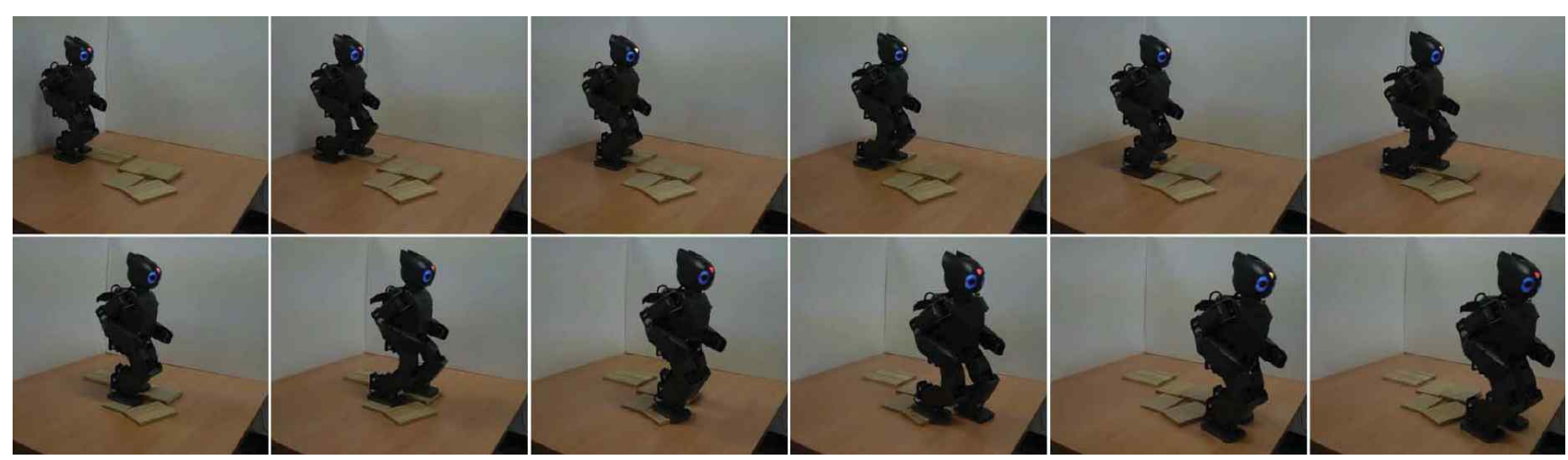

Fig. 5. Snapshots of the walking experiment on unknown uneven terrain (left to right, top to bottom) 
trajectories. Consequently, the proposed method enabled the humanoid robot to walk on the unknown uneven terrain stably by modifying the sagittal and lateral step lengths and
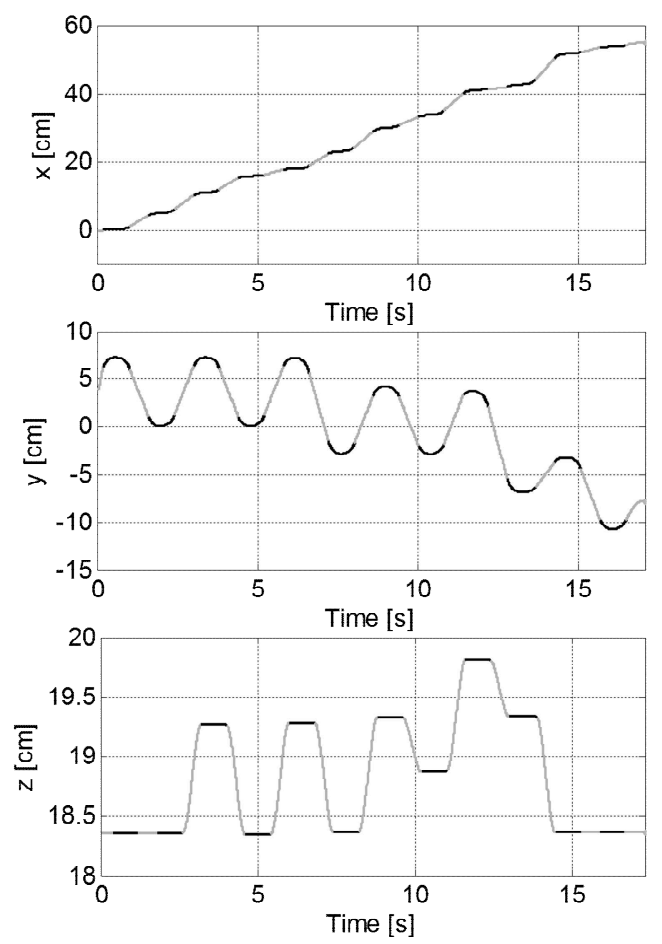

Fig. 6. Generated vertical COM trajectory with respect to the base coordinate frame of the ground. The thick and thin lines represent the COM trajectory in the single- and double-support phases, respectively
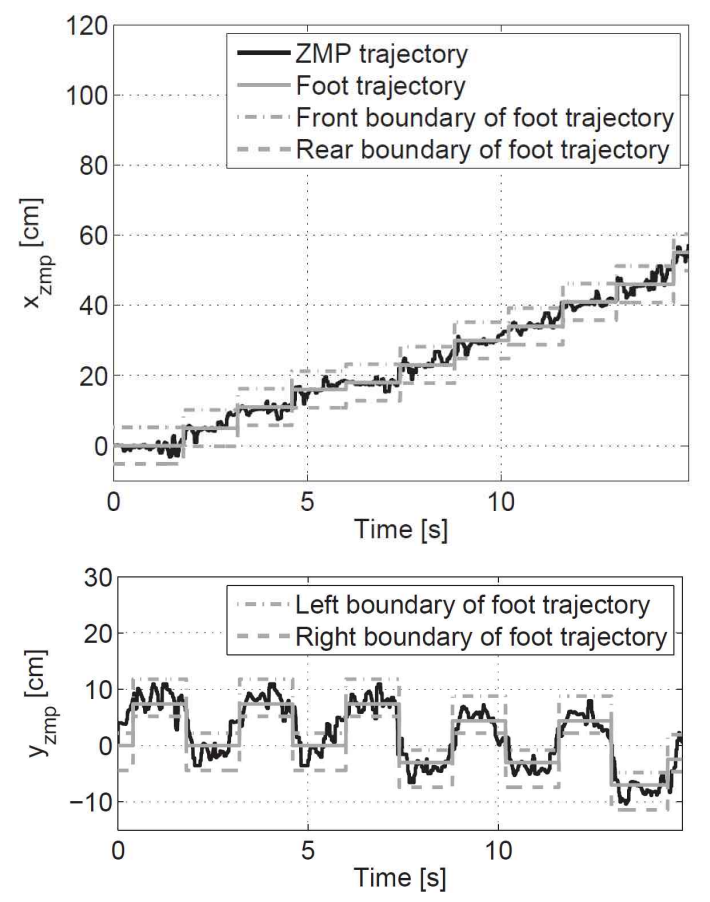

Fig. 7. ZMP trajectories measured in the walking experiment the walking direction at every footstep.

\section{Conclusion}

This paper proposed a method for generating modifiable walking patterns for humanoid robots on unknown uneven terrain. The proposed method uses real-time ZMP variation to generate modifiable walking patterns. Further, the control strategy for walking on unknown uneven terrain is proposed. It compensates for disturbances that occur between the robot and the uneven terrain by using VSD models of the foot. In addition, foot and vertical COM trajectories are generated to realize stable walking on unknown uneven terrain. The effectiveness of the proposed method was demonstrated experimentally. The experimental results indicate that the proposed method enables a humanoid robot to walk stably on uneven terrain, without the knowledge of the exact height of the terrain, by modifying its walking period, sagittal and lateral step lengths, and walking direction at every footstep.

\section{Acknowledgement}

This work was supported by the National Research Foundation of Korea(NRF) grant funded by the Korea government(MSIP) (No. 2016R1C1B1006691 and No. 2014R1A2A1A11053153) and the Ajou University research fund.

\section{References}

[1] S. Kajita, F. Kanehiro, K. Kaneko, K. Fujiwara, K. Yokoi, and H. Hirukawa, "A realtime pattern generator for biped walking," in Proc. IEEE Int. Conf. Robot. Autom., 2002, vol. 1, pp. 31-37.

[2] T. Aoyama, Y. Hasegawa, K. Sekiyama, and T. Fukuda, "Stabilizing and direction control of efficient 3-D biped walking based on PDAC," IEEE/ASME Trans. Mechatron., vol. 14, no. 6, pp. 712-718, Dec. 2009.

[3] K. Harada, S. Hattori, H. Hirukawa, M. Morisawa, S. Kajita, and E. Yoshida, "Two-stage time-parametrized gait planning for humanoid robots," IEEE/ASME Trans. Mechatron., vol. 15, no. 5, pp. 694-703, Oct. 2010.

[4] Y.-D. Hong, B.-J. Lee, and J.-H. Kim, "Command state-based modifiable walking pattern generation on an inclined plane in pitch and roll directions for humanoid robots," IEEE/ASME Trans. Mechatron., vol. 16, no. 4, pp. 783-789, Aug. 2011.

[5] Y. -D. Hong, C. -S. Park, and J. -H. Kim, "Stable bipedal walking with a vertical center of mass motion by an evolutionary optimized central pattern gen- 
erator," IEEE Trans. Ind. Electron., vol. 61, no. 5, pp. 2246-2355, May 2014.

[6] S. Kajita, M. Morisawa, K. Harada, K. Kaneko, F. Kanehiro, K. Fujiwara, and H. Hirukawa, "Biped walking pattern generator allowing auxiliary ZMP control," in Proc. IEEE/RSJ Int. Conf. Intell. Robot. Syst., 2006, pp. 2993-2999.

[7] T. Takubo, Y. Imada, K. Ohara, Y. Mae, and T. Arai, Rough terrain walking for bipedal robot by using ZMP criteria map," in Proc. IEEE Int. Conf. Robot. Autom., 2009, pp. 788-793.

[8] H. Hirukawa, S. Hattori, S. Kajita, K. Harada, K. Kaneko, F. Kanehiro, M. Morisawa, and S. Nakaoka, "A pattern generator of humanoid robots walking on a rough terrain," in Proc. IEEE Int. Conf. Robot. Autom., 2007, pp. 2181-2187.

[9] Y. Zheng, M. C. Lin, D. Manocha, A. H. Adiwahono, and C.-M. Chew, "A walking pattern generator for biped robots on uneven terrains," in Proc. IEEE/RSJ Int. Conf. Intell. Robot. Syst., 2010, pp. 4483-4488.

[10] Q. Huang and Y. Nakamura, "Sensory reflex control for humanoid walking," IEEE Trans. Robot., vol. 21, no. 5, pp. 977-984, Oct. 2005.

[11] K. Nishiwaki and S. Kagami, "Walking control on uneven terrain with short cycle pattern generation," in Proc. IEEE-RAS Int. Conf. Humanoid Robots, 2007, pp. 447-453.

[12] E. Ohashi, T. Sato, and K. Ohnishi, "A walking stabilization method based on environmental modes on each foot for biped robot," IEEE Trans. Ind. Electron., vol. 56, no. 10, pp. 3964-3974, Oct. 2009.

[13] H.-J. Kang, K. Hashimoto, H. Kondo, K. Hattori, K. Nishikawa, Y. Hama, H.-O. Lim, A. Takanishi, K. Suga, and K. Kato, "Realization of biped walking on uneven terrain by new foot mechanism capable of detecting ground surface," in Proc. IEEE Int. Conf. Robot. Autom., 2010, pp. 5167-5172.

[14] Y.-D. Hong and J.-H. Kim, "3-D command statebased modifiable bipedal walking on uneven terrain," IEEE/ASME Trans. Mechatron., vol. 18, no. 2, pp. 657-663, Apr. 2013.

[15] Y.-D. Hong, Y.-H. Kim, J.-H. Han, J.-K. Yoo, and J.-H. Kim, "Evolutionary multiobjective footstep planning for humanoid robots," IEEE Trans. Syst. Man. Cybern. C, Appl. Rev., vol. 41, no. 4, pp. 520532, Jul. 2011.

[16] Y.-D. Hong and J.-H. Kim, “An evolutionary optimized footstep planner for the navigation of humanoid robots," Int. J. Humanoid Robot., vol. 9, no. 1, Mar. 2012.

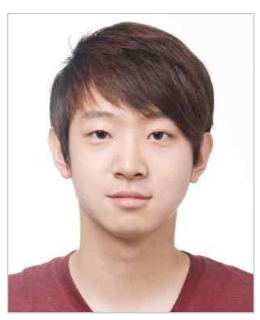

Woong-Ki Lee received the B.S. degree in electrical and computer engineering from Ajou University, Suwon, Korea, in 2015, where he is currently working toward the M.S. degree. His current research interests include humanoid robotics, especially in footstep planning and evolutionary

optimization.

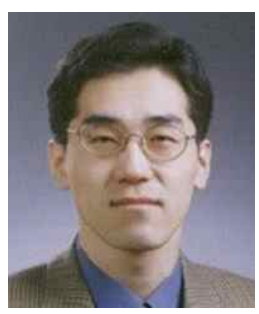

Dongkyoung Chwa received the B.S. and M.S. degrees in control and instrumentation engineering and the Ph.D. degree in electrical and computer engineering from Seoul National University, Seoul, Korea, in 1995, 1997, and 2001, respectively. From 2001 to 2003, he was a Postdoctoral Researcher with Seoul National University, where he was also a BK21 Assistant Professor in 2004. Since 2005, he has been with the Department of Electrical and Computer Engineering, Ajou University, Suwon, Korea, where he is currently a Professor. He was a Visiting Scholar with the University of New South Wales at the Australian Defence Force Academy and the University of Melbourne, Melbourne, Vic., Australia, in 2003 and the University of Florida, Gainesville, in 2011. His research interests include nonlinear, robust, and adaptive control theories and their applications to robotics; underactuated systems, including wheeled mobile robots; underactuated ships; cranes; and guidance and control of flight systems.

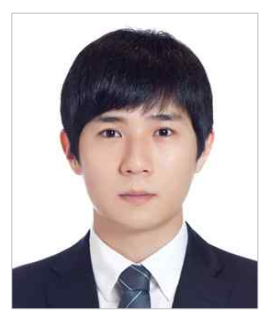

Young-Dae Hong received the B.S., M.S., and Ph.D. degrees in electrical engineering from KAIST, Daejeon, Korea, in 2007, 2009, and 2013, respectively. Since 2014 , he has been with the department of electrical and computer engineering, Ajou University, Suwon, Korea, where he is currently an Assistant Professor. His current research interests include humanoid robotics, especially in bipedal walking pattern generation, footstep planning, multi/single objective evolutionary optimization, and biologically inspired control. 